\title{
Mechanisms of lymphatic metastasis
}

\author{
Sinem Karaman and Michael Detmar
}

\author{
Institute of Pharmaceutical Sciences, Swiss Federal Institute of Technology, ETH Zurich, Zurich, Switzerland.
}

\begin{abstract}
Malignant tumors release growth factors such as VEGF-C to induce lymphatic vessel expansion (lymphangiogenesis) in primary tumors and in draining sentinel LNs, thereby promoting LN metastasis. Surprising recent evidence suggests that lymphatic vessels do not merely represent passive channels for tumor spread, but that they may actively promote tumor cell recruitment to LNs, cancer stem cell survival, and immune modulation. New imaging approaches allow the sensitive visualization of the earliest LN metastases and the quantitative, noninvasive measurement of the function of tumor-draining lymphatic vessels, with potential applications in the development of biomarkers for prognosis and measurement of therapeutic response.
\end{abstract}

Cancer metastasis, the dissemination of cancer cells from the primary tumor to organs, where they initiate malignant growth, is the primary cause of cancer-related deaths. There has been a plethora of studies addressing the mechanisms of tumor metastasis via the bloodstream to distant organs; however, the majority of epithelial cancers first develop metastatic growth by spreading via lymphatic vessels to their draining LNs. Indeed, the detection of metastases within the sentinel LNs (SLNs; the first LNs into which a tumor drains) has major prognostic implications for patient survival and often also determines the choice of adjuvant therapies (1). Despite the obvious clinical importance of LN metastasis, the mechanisms leading to tumor spread via lymphatic vessels have remained unknown for decades. In fact, the prevailing view suggested that lymphatic vessels only play a passive role in tumor metastasis, serving merely as channels for tissue-invading tumor cells. The limited knowledge in this field was due to the relatively low scientific interest in lymphatic vessels as compared to the blood vasculature, the lack of reliable molecular markers to distinguish between lymphatic and blood vessels, the absence of identified growth factors for the lymphatic system, and the paucity of suitable experimental models to study and quantify LN metastasis. During the last 15 years, however, there has been substantial progress in the field of lymphatic vessel biology, which has rapidly lead to the recognition of the lymphatic vascular system as a major player involved in a multitude of human diseases (2). In this article, we will discuss the major discoveries made by our laboratory and many other researchers that have led to the recognition of a major role for the lymphatic vasculature in promoting cancer metastasis and to the new concepts of tumor-associated and LN lymphangiogenesis with a specific focus on the development of new strategies to image and therapeutically target the lymphatic system in cancer.

\section{Identification of lymphatic-specific markers and growth factors}

In the past, studies of lymphatic metastasis have been hampered by the lack of molecular markers that reliably distinguish lymphatic vessels from blood vessels within and surrounding the primary tumors. A major breakthrough was the discovery of the lymphangiogenic growth factors VEGF-C and VEGF-D and their specific receptor, VEGFR-3, and its role in lymphatic development (2-4). The discovery of the lymphatic-specific marker lymphatic vessel endothelial hyaluronan receptor 1 (LYVE1) in 1999 (5) pro-

Conflict of interest: The authors have declared that no conflict of interest exists. Citation for this article: J Clin Invest. 2014;124(3):922-928. doi:10.1172/JCI71606. vided a powerful tool to specifically identify lymphatic vessels in a variety of tissues and opened up the path to detailed investigations of tumor-associated lymphatics (6-8). The transcription factor prospero-related homeobox 1 (PROX1) was also found to be specifically expressed by lymphatic but not blood vascular endothelium (9). In addition, the discovery that the membrane glycoprotein podoplanin is specifically expressed by lymphatic but not blood vessels (10), and the recognition that a commercially available antibody named D2-40 (which was originally raised against an oncofetal antigen in testicular germ cell tumors; ref. 11) specifically detects human podoplanin (12), facilitated widespread investigations of lymphatic vessel involvement in human cancers.

\section{Tumor lymphangiogenesis}

In 2001, three groups independently identified a new mechanism of tumor metastasis, namely the induction of tumor lymphangiogenesis, by combining the use of the new lymphatic marker LYVE1 with overexpression of VEGF-C or VEGF-D in experimental tumor models (6-8). In these studies, overexpression of VEGF-C or VEGF-D promoted the growth of tumor-associated lymphatic vessels and also enhanced LN metastasis (Figure 1). These findings contributed to a dramatically increased scientific interest in lymphatic biology; however, their impact was initially controversial, since it was unclear whether this mechanism might be restricted to mouse models of cancer with unproven relevance for human cancer progression. Thus, a series of retrospective studies was initiated to evaluate the prognostic relevance of tumor lymphangiogenesis in different types of human cancer (13). In head and neck cancer, a correlation between intratumoral lymphatic vessel density and LN metastasis was found (14). Quantification of intratumoral and peritumoral lymphatic vessels in primary human malignant melanomas of the skin revealed that tumor lymphangiogenesis indeed represented a novel prognostic indicator for metastasis and overall patient survival (15). In a follow-up study, the extent of lymphangiogenesis in primary melanomas was the most significant predictor of the presence of SLN metastases at the time of surgery, with a higher significance than tumor thickness (16). Since then, a large number of clinical studies have investigated the correlation of LN metastasis with tumor lymphangiogenesis or with tumor expression of the lymphangiogenic factors VEGF-C and VEGF-D in a variety of human cancers (reviewed in ref. 13). A correlation between VEGF-C or VEGF-D expression in primary tumors and $\mathrm{LN}$ metastasis was found in cancers of the breast, colon, rectum, prostate, esophagus, stomach, lung, uterine cervix, endometrium, and other tissues $(17,18)$. 
A

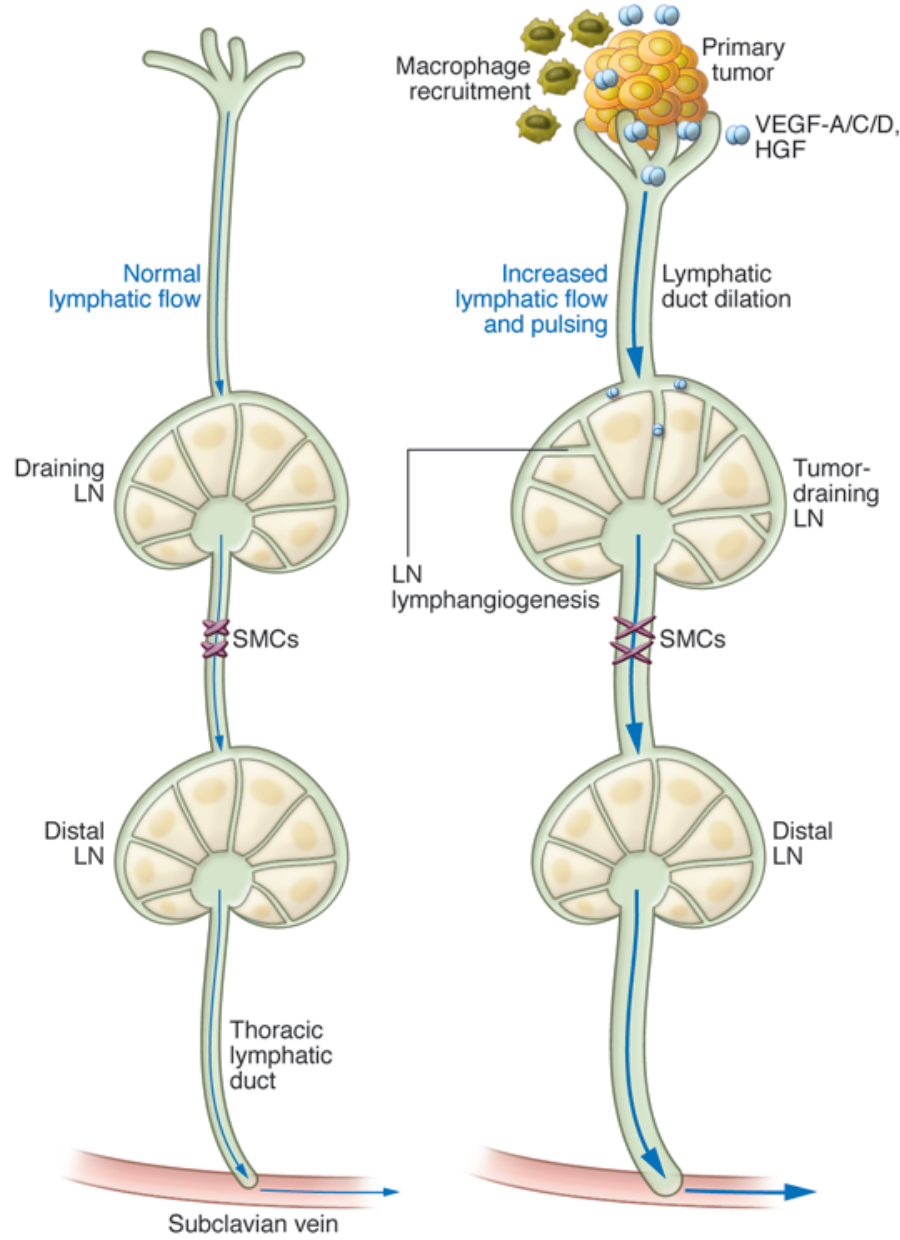

C
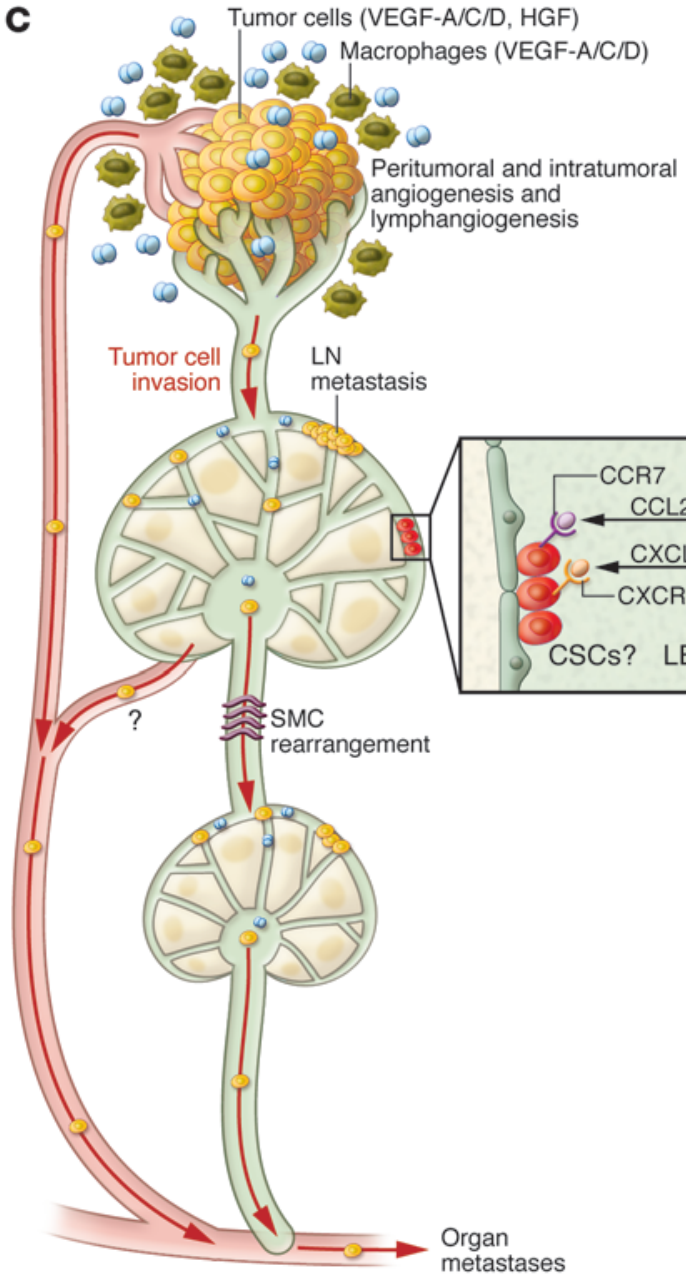

Figure 1

An important contribution of tumor and LN lymphangiogenesis to cancer metastasis. (A) Normal lymphatic tissue drainage through lymphatic capillaries, collecting lymphatics, and LNs. (B) Lymphangiogenic factors produced by premetastatic tumors, including VEGF-C, VEGF-D, VEGF-A, and HGF, are taken up by peritumoral lymphatic capillaries and are transported via the collecting lymphatics toward the tumor-draining SLN, where they act directly on preexisting lymphatic vessels to induce LN lymphangiogenesis. Tumor-draining lymphatic vessels display an enlarged size and increased lymph flow and pulsing. (C) Once metastatic tumor cells have spread to their draining LNs, they serve as a major source of lymphangiogenic factors. These promote the remodeling and SMC rearrangement of distant (post-SLN) lymphatic vessels and lymphangiogenesis in distant LNs and promote secondary metastasis, including organ metastasis, via the thoracic duct, which connects to the venous circulation via the subclavian vein. CSC, cancer stem cell. The chemokines CCL21 and CXCL12, released by activated lymphatic endothelial cells (LECs) within SLNs, might provide a niche for cancer cells with stem cell-like properties that express the receptors CCR7 and CXCR4.

A majority of studies confirmed the concept that tumor-induced lymphangiogenesis and increased expression of lymphangiogenic growth factors is associated with increased rates of $\mathrm{LN}$ metastasis and with poor prognosis $(13,19)$. In addition to members of the VEGF family of growth factors, a number of other mediators have also been implicated in promoting lymphangiogenesis in tumors and other conditions, including PDGF-BB (20), IGF1 and -2 (21), FGF2 (22-26), HGF $(27,28)$, angiopoietin-2 (29), sphingosine-1phosphate (30), adrenomedullin (31), and IL-7 (32, 33). The relative importance of these factors compared with the VEGFs remains to be investigated in individual cancer types. The cellular and molecular mediation of lymphangiogenesis during early tumor development has not been characterized in detail. Experimental evidence indicates, however, that the tumor-induced lym- phatic vessels sprout from preexisting lymphatic networks, and that there is little if any contribution from bone marrow-derived endothelial progenitor cells (34).

\section{LN lymphangiogenesis}

Activation of the lymphatic vasculature is not restricted to the primary tumor microenvironment. Using a multistep chemical skin carcinogenesis regimen in mice with transgenic overexpression of VEGF-A, we found that active lymphangiogenesis also occurred in metastatic tumor-draining LNs (35). Importantly, LN lymphangiogenesis could be detected in tumor-draining LNs prior to the actual onset of lymphatic metastasis, indicating the creation of a premetastatic niche by the primary tumors (35). Further studies in mice with transgenic overexpression of VEGF-C in the skin revealed that 
A

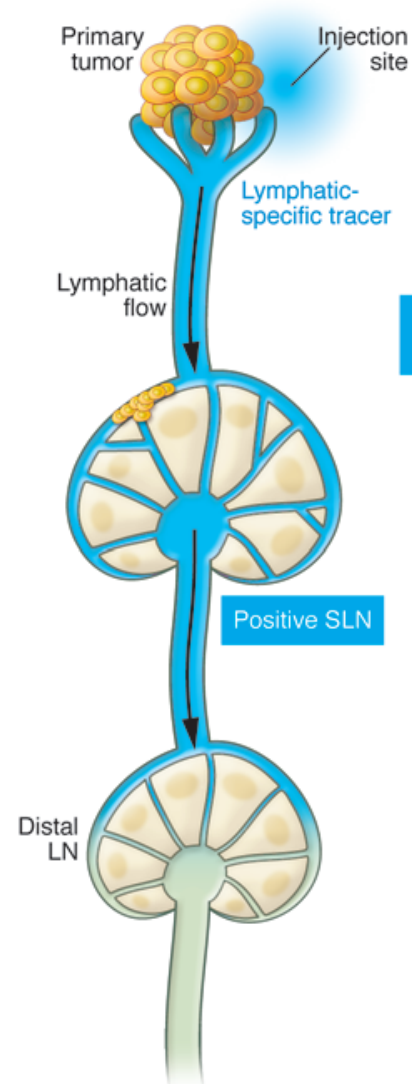

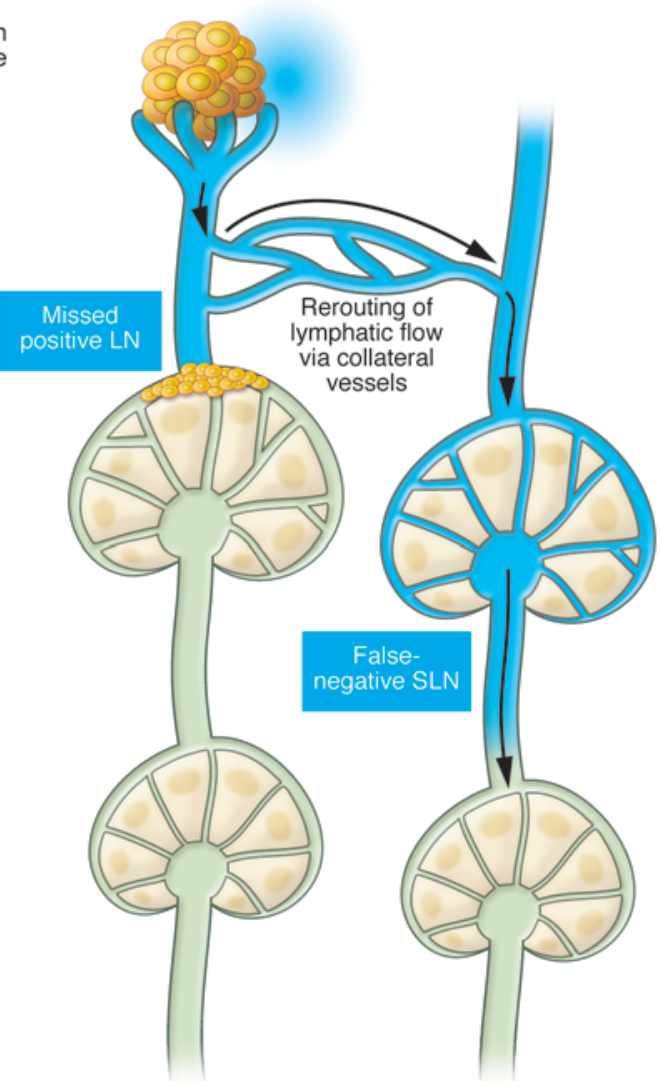

C

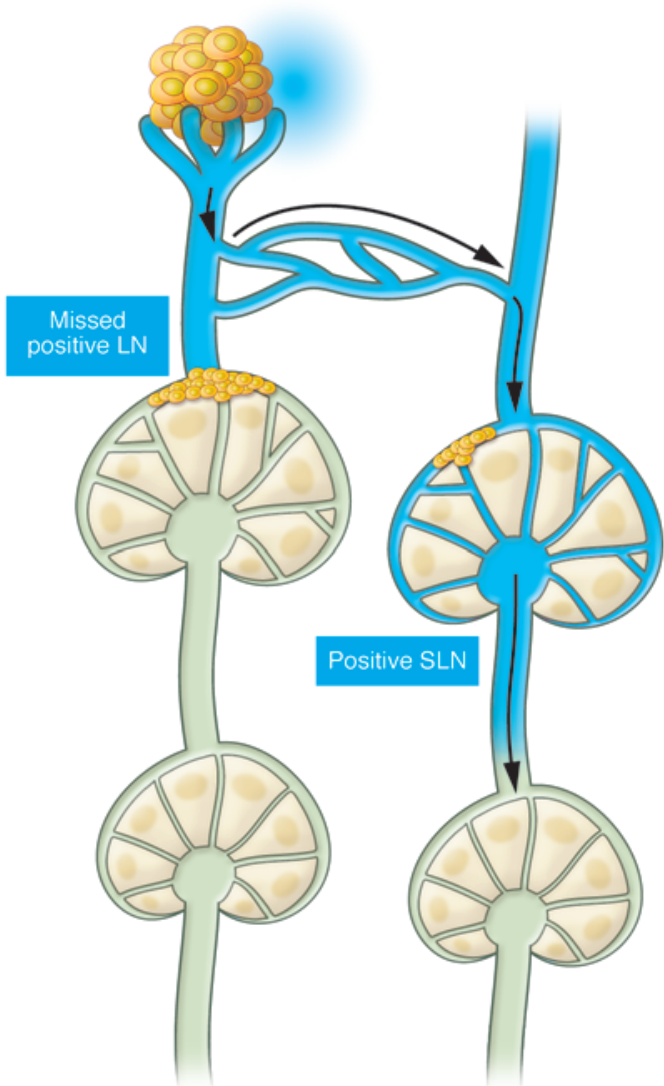

Figure 2

SLN metastases can impair lymphatic drainage and lead to rerouting of lymphatic flow. (A) After peritumoral injection around the primary tumor, the lymphatic tracer is taken up by lymphatic capillaries and is transported via collecting lymphatics to the draining SLN, which may contain metastasized tumor cells (positive SLN) or not (negative SLN). (B) Larger metastases within the SLN can obstruct the lymphatic drainage, leading to rerouting of lymph flow toward another LN that may be metastasis free and therefore may represent a false-negative SLN, leading to incorrect cancer staging. (C) At later stages, this LN may also contain metastatic tumor cells (positive SLN).

tumor-induced LN lymphangiogenesis promoted further metastasis to distant LNs and beyond to distant organs (36). While these findings were initially restricted to experimental mouse models of cancer, LN lymphangiogenesis was subsequently also detected within SLNs in human cancers, including cutaneous malignant melanoma, breast cancer, and extramammary Paget's disease (16, 37-39). Moreover, the extent of SLN lymphangiogenesis, lipoxygenase expression, and lymphatic tumor invasion was correlated with non-SLN metastasis in breast cancer patients $(38,40)$.

The mechanisms mediating lymphatic vessel expansion within tumor-draining LNs are incompletely understood. Most likely, lymphangiogenic factors produced by the primary tumor, including VEGF-C, VEGF-D, VEGF-A, and HGF, are taken up by peritumoral lymphatic capillaries and are transported via the collecting lymphatics toward the SLNs, where they act directly on preexisting lymphatic vessels (Figure 1, A and B), similar to inflammation-induced lymphangiogenesis (41). This concept is supported by the observed changes within tumor-draining lymphatic vessels that include enlarged size and increased lymph flow and pulsing (Figure 1B and refs. 42-44). It remains to be investigated whether tumor-released exosomes $(45,46)$ and local pro- duction of lymphangiogenic factors within tumor-draining LNs, by macrophages (47) or other cell types, might further contribute to LN lymphangiogenesis. In particular, B cells and B cell-derived VEGF have been implicated in promoting LN lymphangiogenesis in inflammation and cancer draining LNs (48-50). Once metastatic tumor cells have spread to their draining LNs, they represent a major source of lymphangiogenic factors such as VEGF-C (38), contributing to the recently observed remodeling and SMC rearrangement of distant (post-SLN) lymphatic vessels and LNs, and secondary metastasis including organ metastasis via the thoracic duct, which connects to the subclavian vein and thus to the general circulation (Figure $1 \mathrm{C}$ and ref. 51).

\section{Lymphatic vessel effects on metastatic tumor cells}

The traditional concept of a merely passive role of lymphatic vessels in cancer metastasis has been challenged recently. An increased number and size of peritumoral lymphatic vessels might provide more opportunities for cancer cell intravasation (lymphatic invasion), but tumor-draining lymphatics might promote tumor spread also by increased pumping and lymph flow, often mediated by VEGF-C $(44,49,52)$. In addition, lymphatic vessels make 
several key contributions to metastasis, which likely include the targeted recruitment of cancer cells toward lymphatic vessels and LNs, the provision of a cancer stem cell niche, and the modulation of antitumor immune responses at the level of the primary tumor and the metastatic LN. Within most non-neoplastic tissues, lymphatic vessels are a major source of the chemokine CCL21, which binds to the CCR7 receptor on activated DCs, thereby recruiting them toward the lymphatic vasculature and finally toward the draining LNs to initiate immune responses (53). In in vitro studies, enhanced lymphatic flow has been found to upregulate CCL21 production by the lymphatic endothelium (54). CCR7 expression has also been detected on a variety of human cancer cells and has been correlated with increased metastasis (55). Importantly, LN metastasis was greatly enhanced in an experimental tumor model when transplanted melanoma cells overexpressed CCR7 (56), indicating that lymphatic endothelium may provide guidance cues for metastatic cancer cells that are normally used for the physiologic function of the immune system. More recently, an important role of the chemokine CXCL12 (stromal-derived factor 1) in lymphatic cancer metastasis of extramammary Paget's disease has been suggested. CXCL12 expression was upregulated in tumor-associated lymphatic endothelium and in the lymphangiogenic endothelium in the subcapsular sinuses of tumor-draining LNs, whereas its receptor, CXCR4, was expressed by invading tumor cells (37). In addition, CXCR3 expression on tumor cells has also been correlated with enhanced rates of LN metastases (57), but future studies are needed to evaluate the relative contribution of lymphatic endothelium toward the production of CXCR3 ligands. More recently, an important role of chemokines in mediating tumor cell entry into LNs has been identified. The CCL1 chemokine, produced by LN lymphatic sinuses, mediated the LN entry of CCR8-expressing melanoma cells, whereas CCR8 blockade reduced LN metastasis (58). In particular, CCR8 inhibition resulted in an arrest of tumor cells in the collecting lymphatic vessels, at the junction with the subcapsular LN sinus (58).

Lymphatic endothelium might also provide a niche for cancer cells with stem cell-like properties (Figure 1C). Clinical observations of so-called "in-transit metastases," i.e., metastatic tumors that develop in lymphatic vessels between the primary tumor and the draining LN, have suggested that lymphatic endothelium might provide a protective microenvironment for long-term tumor cell survival. Moreover, tumor cells might remain dormant within draining LNs for extended periods of time after removal of the primary tumor (59). Recently, it was found that CXCR4+ melanoma cells, which also expressed the stem cell marker CD133, were located close to CXCL12-producing lymphatic vessels in metastatic LNs and lungs (60). CXCR $4^{+} / \mathrm{CD} 133^{+}$cells had a higher metastatic activity than CXCR4-/CD133+ cells. Additionally, CXCR4 blockade together with the alkylating agent dacarbazine, which is widely used to treat human melanomas, but not dacarbazine treatment alone, potently inhibited melanoma growth and metastasis (60). In addition to these direct effects of lymphatic endothelium on cancer cell survival, lymphatic vessels might also provide an immunoprotective microenvironment via chemokine secretion. Recent studies with CCL21-expressing tumors indicate that CCL21 might shift the host immune response from immunogenic to tolerogenic, which could potentially facilitate tumor progression (61). Moreover, activation of LN lymphatic vessels by VEGF-C was reported to induce immune tolerance in a melanoma model (62). A major role of lymphatic endothelial cells in mediating T cell tolerance via PD-L1 was recently identified (63-65). These findings are in line with the recently discovered antiinflammatory effect of VEGF-C-mediated activation of lymphatic endothelium in models of cutaneous inflammation and rheumatoid arthritis (66-69), and they reveal an unanticipated role for lymphatic vessels in actively shaping immune responses (70).

\section{In vivo imaging of LN lymphangiogenesis for early metastasis detection}

Based on the extensive expansion of the lymphatic vasculature in tumor-draining LNs, even at very early stages of metastasis when direct visualization of tumor cells by standard methods might not yet be feasible, imaging of LN lymphangiogenesis might represent a potential strategy for the early detection of metastases. Recent studies in experimental mouse melanomas indicate that an intravenously injected antibody against the lymphatic marker LYVE1 specifically accumulates within 24 hours in tumor-draining, metastasis-containing LNs, as evaluated in biodistribution studies and using immunodetection, which revealed specific targeting to the expanded lymphatic vasculature (71). Use of a ${ }^{124}$ I-radiolabeled anti-LYVE1 antibody and immuno-PET enabled the noninvasive detection of LN metastases of primary melanomas in mice, based on stromal lymphatic activation $(71,72)$. Importantly, this immunoPET approach detected early LN metastases more sensitively than standard PET imaging using $\left[{ }^{18} \mathrm{~F}\right]$ fluorodeoxyglucose (71). Although inflammation-draining LNs are also characterized by lymphatic expansion $(41,48,73)$, these changes are reversible (74). Further studies are needed to evaluate the specificity of the immunoPET approach in more detail.

\section{Functional, quantitative in vivo imaging of tumor-draining lymphatic vessels}

Visualization of tumor-draining lymphatic vessels and LNs, in particular of the first draining LN (SLN), is frequently pursued during surgical resection of primary melanomas and breast cancers, using peritumoral tracer injection (Figure $2 \mathrm{~A}$ and refs. 75,76 ). However, these methods do not provide sufficient information about the functionality of the entire draining lymphatic network. Based on the experimental and clinical evidence for an important role of the lymphatic vasculature in tumor progression, there is a great need for the identification of biomarkers to evaluate the functional status of tumor-associated lymphatic vessels and to quantitatively measure the efficacy of antilymphangiogenic therapies. Imaging in the near-infrared range provides promising opportunities in this regard, based on the low tissue absorption and low autofluorescence in this wavelength range (77). We previously developed liposomes that contain the near-infrared dye indocyanine green (ICG), which has been used in its free form for lymphatic imaging in humans (78). Liposomal encapsulation increased the brightness and stability of ICG and also resulted in specific uptake by lymphatic vessels after intradermal injection (44). In vivo imaging using liposomal ICG in a footpad melanoma model enabled the quantitation of lymphatic flow through draining LNs and revealed that lymph flow was increased in tumor-draining lymphatic vessels, as compared with normal tissue lymph flow, and that overexpression of VEGF-C further promoted lymph flow (44).

In a further improvement of tracer development, PEG conjugates of IRDyes, a class of much brighter near-infrared dyes, were developed, which enabled in vivo imaging with excellent spatial and temporal resolution of initial and collecting lymphatic vessels 
$(77,79,80)$. In vivo near-infrared imaging of experimental tumors with PEG-conjugated dyes enabled the dynamic noninvasive visualization of tumor-draining lymphatic vessels and also revealed that the valves in the enlarged tumor-draining, collecting lymphatic vessels remain functional despite the increased fluid load (80). This method also allowed for the noninvasive quantification of the frequency of lymphatic vessel contractions and thus of the functional effects of specific drugs (81). Repeated imaging of tumorbearing mice over several weeks revealed that at a certain size of SLN metastasis, the flow through the SLN was obstructed, leading to a rerouting of lymph flow from the tumor to newly developed collateral lymphatic vessels that drained to different LNs (Figure 2 and ref. 80). These experimental findings are in agreement with the clinically observed redirection of lymph flow after LN metastasis of penile cancers (82). Bypassing of metastatic LNs with increased intranodal pressure toward the next echelon of nodes with lower intranodal pressure has also been reported in breast cancer (83). A similar rerouting of lymph flow was also found after surgical LN resection (84). These findings have clinical relevance, as lymphatic rerouting may contribute to misleading results of SLN dissections during cancer surgery (81).

The mechanisms that mediate lymphatic rerouting are not fully understood. Recent studies indicate, however, that increased fluid load might promote VEGFR-3 phosphorylation, lymphatic endothelial cell proliferation and lymphatic vessel generation $(85,86)$. After peritumoral injection around the primary tumor, the lymphatic tracer is taken up by lymphatic capillaries and is transported via collecting lymphatics to the draining SLN that may contain tumor metastasis (positive SLN; Figure 2A) or not (negative SLN). Larger metastases within the SLN can obstruct the lymphatic drainage, rerouting the lymph flow toward another LN that may be metastasis free. This scenario could produce a falsenegative SLN (Figure 2B), leading to an incorrect staging of the cancer patient. At later stages, this LN may contain metastatic tumor cells as well (positive SLN, Figure 2C).

\section{Perspectives and open questions}

There is now convincing evidence that tumor lymphangiogenesis and LN lymphangiogenesis represent important prognostic markers for the risk of future metastasis and overall survival in an increasing number of tumors, including malignant melanomas and epithelial cancers (19). Thus, it might seem appealing to include the quantitative determination of lymphatic vessel expansion within primary tumors and SLNs in routine pathological examinations. However, there are at present no standardized methods established for the reproducible quantification of lymphatic vessels in tissue sections, despite previous efforts in this regard (87). The use of antibodies against the lymphatic marker podoplanin represents a reliable approach for the identification of lymphatic vessels in most human tissues, although one has to keep in mind that podoplanin is also expressed by several nonvascular cells including myoepithelial cells (88). LYVE1 is also expressed on some types of blood vessels and might be modulated by inflammation $(89,90)$, but has been successfully used to analyze lymphatic vessels in many types of cancer. In contrast, the nuclear PROX1 stain is often difficult to distinguish, and antibodies to PROX1 should be combined with antibodies to other lymphatic markers or to the panvascular marker CD31. Since quantification based on immunostaining is time-consuming and possibly observer dependent, semiquantitative evaluation of the presence of intratumoral lymphatics or of lymphatic vessel invasion might represent a more practical and feasible approach (91). Thus, large-scale prospective clinicopathological studies need to be initiated to validate the prognostic relevance of such approaches.

Based on its potential contribution to cancer spread, immune evasion by cancer cells, and the maintenance of cancer stem cell-like properties, the lymphatic endothelium has become an attractive therapeutic target for new cancer therapies, including photodynamic ablation of lymphatic vessels (92). Therapies aimed at blocking the VEGF-C/VEGF-D/VEGFR-3 pathway have entered clinical trials and could potentially also reduce blood vascular angiogenesis in certain types of tumors $(93,94)$. However, several additional lymphangiogenic pathways have been identified (19), and phenotypic chemical genetic screens in Xenopus embryos and in 3-dimensional human lymphatic endothelial spheroids have identified a range of compounds, including drugs already approved for clinical use, that reduce lymphatic vessel growth in vivo $(25,95)$. One remaining challenge is the identification of rational combination regimens of antilymphangiogenic drugs with established chemotherapies and targeted anticancer therapies. It has remained controversial whether LN metastases represent mediators of systemic metastasis to distant organs or whether they are mere indicators of cancer aggressiveness (96). Thus, there is a clear need for more sophisticated experimental models to label and track the fate of cancer cells once they have reached the draining LN. Moreover, new technologies need to be applied to spatiotemporally dissect the successive steps of LN metastasis, ranging from the early steps of tumor cell entry from the LN lymphatic sinuses (58) to the potential tumor cell exit routes, including the detailed cellular interactions with distinct LN subpopulations.

The recent development of new, noninvasive in vivo imaging techniques might lead to potential clinical applications regarding the early detection of metastases based on the visualization of tumor-induced stromal changes, such as lymphatic vessel expansion in SLNs, instead of the tumor cells themselves. Identification of specific biomarkers for the reliable targeting of tumor-activated lymphatic endothelium, using genomic (97) or proteomic techniques (98), currently represents a major challenge. A study using transcriptional profiling of cultured, tumor-derived lymphatic endothelial cells revealed increased expression of functionally relevant molecules, including the tight junction regulatory protein endothelial-specific adhesion molecule (ESAM) and the TGF- $\beta$ coreceptor endoglin (CD105) (97). ESAM expression in tumor lymphatics was correlated with nodal metastasis of head and neck squamous cell carcinomas and colorectal carcinomas $(97,99)$. Future studies are needed to investigate the transcriptional activity and the proteome of directly isolated tumor-associated lymphatic endothelium. The establishment of new, near-infrared tracers for the quantitative, noninvasive, in vivo imaging of lymphatic vessel function now provides the opportunity to study lymphatic function as a biomarker for the response to antilymphangiogenic and other therapies. The development of lymphedema is a major complication of breast cancer surgery and even SLN dissection (19) that occurs over the course of several years following surgical excision of the primary tumor. Since lymphatic vessels can efficiently regenerate when challenged by increased fluid load $(84,86)$, it is tempting to speculate that preoperative quantitative measurement of lymphatic vessel function in the arms might identify those patients at risk for postsurgical lymphedema, enabling the imme- 
diate onset of measures such as pneumatic compression, which may result in increased tissue fluid pressure up to $100 \mathrm{mmHg}$ (100), or other physical stimulations of lymphatic function to prevent lymphedema formation.

\section{Acknowledgments}

Work in the authors' laboratory is supported by the Swiss National Foundation (grant 310030B-137087), the European Research Council (grant LYVICAM), the Leducq Foundation

1. de Boer M, van Dijck JA, Bult P, Borm GF, TjanHeijnen VC. Breast cancer prognosis and occult lymph node metastases, isolated tumor cells, and micrometastases. J Natl Cancer Inst. 2010; 102(6):410-425.

2. Tammela T, Alitalo K. Lymphangiogenesis: Molecular mechanisms and future promise. Cell. 2010; 140(4):460-476.

3. Kaipainen A, et al. Expression of the fms-like tyrosine kinase 4 gene becomes restricted to lymphatic endothelium during development. Proc Natl Acad Sci US A. 1995;92(8):3566-3570.

4. Kukk E, et al. VEGF-C receptor binding and pattern of expression with VEGFR-3 suggests a role in lymphatic vascular development. Development. 1996; 122(12):3829-3837.

5. Banerji S, et al. LYVE-1, a new homologue of the CD44 glycoprotein, is a lymph-specific receptor for hyaluronan. J Cell Biol. 1999;144(4):789-801.

6. Mandriota SJ, et al. Vascular endothelial growth factor-C-mediated lymphangiogenesis promotes tumour metastasis. EMBO J. 2001;20(4):672-682.

7. Skobe $M$, et al. Induction of tumor lymphangiogenesis by VEGF-C promotes breast cancer metastasis. Nat Med. 2001;7(2):192-198.

8. Stacker SA, et al. VEGF-D promotes the metastatic spread of tumor cells via the lymphatics. Nat Med. 2001;7(2):186-191.

9. Wigle JT, Oliver G. Prox1 function is required for the development of the murine lymphatic system. Cell. 1999;98(6):769-778.

10. Breiteneder-Geleff S, et al. Angiosarcomas express mixed endothelial phenotypes of blood and lymphatic capillaries: podoplanin as a specific marker for lymphatic endothelium. Am J Pathol. 1999; 154(2):385-394.

11. Marks A, et al. Characterization and distribution of an oncofetal antigen (M2A antigen) expressed on testicular germ cell tumours. Br J Cancer. 1999; 80(3-4):569-578.

12. Schacht V, Dadras SS, Johnson LA, Jackson DG, Hong YK, Detmar M. Up-regulation of the lymphatic marker podoplanin, a mucin-type transmembrane glycoprotein, in human squamous cell carcinomas and germ cell tumors. Am J Pathol. 2005; 166(3):913-921.

13. Rinderknecht M, Detmar M. Molecular mechanisms of lymph node metastasis. In: Stacker SA, Achen MG, eds. Lymphangiogenesis In Cancer Metastasis. Vol. 13. Berlin, Germany: Springer; 2009:55-82.

14. Beasley NJ, et al. Intratumoral lymphangiogenesis and lymph node metastasis in head and neck cancer. Cancer Res. 2002;62(5):1315-1320.

15. Dadras SS, et al. Tumor lymphangiogenesis: a novel prognostic indicator for cutaneous melanoma metastasis and survival. Am J Pathol. 2003; 162(6):1951-1960.

16. Dadras SS, et al. Tumor lymphangiogenesis predicts melanoma metastasis to sentinel lymph nodes. Mod Pathol. 2005;18(9):1232-1242.

17. Jain RK, Fenton BT. Intratumoral lymphatic vessels: a case of mistaken identity or malfunction? J Natl Cancer Inst. 2002;94(6):417-421.

18. Nathanson SD. Insights into the mechanisms of lymph node metastasis. Cancer. 2003;98(2):413-423.
19. Alitalo A, Detmar M. Interaction of tumor cells and lymphatic vessels in cancer progression. Oncogene. 2012;31(42):4499-4508.

20. Cao R, et al. PDGF-BB induces intratumoral lymphangiogenesis and promotes lymphatic metastasis. Cancer Cell. 2004;6(4):333-345.

21. Bjorndahl $\mathrm{M}$, et al. Insulin-like growth factors 1 and 2 induce lymphangiogenesis in vivo. Proc Natl Acad Sci U S A. 2005;102(43):15593-15598.

22. Chang LK, et al. Dose-dependent response of FGF-2 for lymphangiogenesis. Proc Natl Acad Sci US A. 2004;101(32):11658-11663.

23. Kubo H, Cao R, Brakenhielm E, Makinen T, Cao Y, Alitalo K. Blockade of vascular endothelial growth factor receptor-3 signaling inhibits fibroblast growth factor-2-induced lymphangiogenesis in mouse cornea. Proc Natl Acad Sci U S A. 2002; 99(13):8868-8873.

24. Platonova $\mathrm{N}$, et al. Evidence for the interaction of fibroblast growth factor- 2 with the lymphatic endothelial cell marker LYVE-1. Blood. 2013; 121(7):1229-1237.

25. Schulz MM, et al. Phenotype-based high-content chemical library screening identifies statins as inhibitors of in vivo lymphangiogenesis. Proc Natl Acad Sci U S A. 2012;109(40):E2665-E2674.

26. Shin JW, et al. Prox1 promotes lineage-specific expression of fibroblast growth factor (FGF) receptor-3 in lymphatic endothelium: a role for FGF signaling in lymphangiogenesis. Mol Biol Cell. 2006; 17(2):576-584.

27. Cao R, et al. Hepatocyte growth factor is a lymphangiogenic factor with an indirect mechanism of action. Blood. 2006;107(9):3531-3536.

28. Kajiya K, Hirakawa S, Ma B, Drinnenberg I, Detmar M. Hepatocyte growth factor promotes lymphatic vessel formation and function. EMBO J. 2005;24(16):2885-2895

29. Holopainen T, et al. Effects of angiopoietin-2blocking antibody on endothelial cell-cell junctions and lung metastasis. J Natl Cancer Inst. 2012; 104(6):461-475.

30. Nagahashi M, et al. Sphingosine-1-phosphate produced by sphingosine kinase 1 promotes breast cancer progression by stimulating angiogenesis and lymphangiogenesis. Cancer Res. 2012;72(3):726-735.

31. Karpinich NO, Kechele DO, Espenschied ST, Willcockson HH, Fedoriw Y, Caron KM. Adrenomedullin gene dosage correlates with tumor and lymph node lymphangiogenesis. FASEB J. 2013;27(2):590-600.

32. Iolyeva $M$, et al. Interleukin-7 is produced by afferent lymphatic vessels and supports lymphatic drainage. Blood. 2013;122(13):2271-2281.

33. Jian M, Qingfu Z, Yanduo J, Guocheng J, Xueshan Q. Anti-lymphangiogenesis effects of a specific antiinterleukin 7 receptor antibody in lung cancer model in vivo [published online ahead of print September 24, 2013]. Mol Carcinog. doi:10.1002/mc.22082.

34. He Y, et al. Preexisting lymphatic endothelium but not endothelial progenitor cells are essential for tumor lymphangiogenesis and lymphatic metastasis. Cancer Res. 2004;64(11):3737-3740.

35. Hirakawa S, Kodama S, Kunstfeld R, Kajiya K, Brown LF, Detmar M. VEGF-A induces tumor and sentinel lymph node lymphangiogenesis and promotes lymphatic metastasis. J Exp Med. 2005;
201(7):1089-1099.

36. Hirakawa S, Brown LF, Kodama S, Paavonen K, Alitalo K, Detmar M. VEGF-C-induced lymphangiogenesis in sentinel lymph nodes promotes tumor metastasis to distant sites. Blood. 2007; 109(3):1010-1017.

37. Hirakawa S, et al. Nodal lymphangiogenesis and metastasis: Role of tumor-induced lymphatic vessel activation in extramammary Paget's disease. Am J Pathol. 2009;175(5):2235-2248.

38. Kerjaschki D, et al. Lipoxygenase mediates invasion of intrametastatic lymphatic vessels and propagates lymph node metastasis of human mammary carcinoma xenografts in mouse. J Clin Invest. 2011; 121(5):2000-2012.

39. Van den Eynden GG, et al. Induction of lymphangiogenesis in and around axillary lymph node metastases of patients with breast cancer. BrJ Cancer. 2006;95(10):1362-1366.

40. Van den Eynden GG, et al. Increased sentinel lymph node lymphangiogenesis is associated with nonsentinel axillary lymph node involvement in breast cancer patients with a positive sentinel node. Clin Cancer Res. 2007;13(18 pt 1):5391-5397.

41. Halin C, Tobler NE, Vigl B, Brown LF, Detmar M. VEGF-A produced by chronically inflamed tissue induces lymphangiogenesis in draining lymph nodes. Blood. 2007;110(9):3158-3167.

42. He Y, et al. Vascular endothelial cell growth factor receptor 3-mediated activation of lymphatic endothelium is crucial for tumor cell entry and spread via lymphatic vessels. Cancer Res. 2005;65(11):4739-4746.

43. Hoshida T, et al. Imaging steps of lymphatic metastasis reveals that vascular endothelial growth factor-C increases metastasis by increasing delivery of cancer cells to lymph nodes: therapeutic implications. Cancer Res. 2006;66(16):8065-8075.

44. Proulx ST, et al. Quantitative imaging of lymphatic function with liposomal indocyanine green. Cancer Res. 2010;70(18):7053-7062

45. Hood JL, San RS, Wickline SA. Exosomes released by melanoma cells prepare sentinel lymph nodes for tumor metastasis. Cancer Res. 2011;71(11):3792-3801.

46. Peinado $\mathrm{H}$, et al. Melanoma exosomes educate bone marrow progenitor cells toward a pro-metastatic phenotype through MET. Nat Med. 2012;18(6):883-891.

47. Kerjaschki D. The crucial role of macrophages in lymphangiogenesis. JClin Invest. 2005;115(9):2316-2319.

48. Angeli V, et al. B cell-driven lymphangiogenesis in inflamed lymph nodes enhances dendritic cell mobilization. Immunity. 2006;24(2):203-215.

49. Harrell MI, Iritani BM, Ruddell A. Tumor-induced sentinel lymph node lymphangiogenesis and increased lymph flow precede melanoma metastasis. Am J Pathol. 2007;170(2):774-786.

50. Ruddell A, Mezquita P, Brandvold KA, Farr A, Iritani BM. B lymphocyte-specific c-Myc expression stimulates early and functional expansion of the vasculature and lymphatics during lymphomagenesis. Am J Pathol. 2003;163(6):2233-2245.

51. Gogineni A, et al. Inhibition of VEGF-C modulates distal lymphatic remodeling and secondary metastasis. PloS One. 2013;8(7):e68755.

52. Breslin JW, Gaudreault N, Watson KD, Reynoso 
R, Yuan SY, Wu MH. Vascular endothelial growth factor-C stimulates the lymphatic pump by a VEGF receptor-3-dependent mechanism. Am J Physiol. 2007;293(1):H709-H718.

53. Aebischer D, Iolyeva M, Halin C. The inflammatory response of lymphatic endothelium [published online ahead of print October 24, 2013]. Angiogenesis. doi:10.1007/s10456.013.9404-3.

54. Miteva DO, Rutkowski JM, Dixon JB, Kilarski W, Shields JD, Swartz MA. Transmural flow modulates cell and fluid transport functions of lymphatic endothelium. Circ Res. 2010;106(5):920-931.

55. Muller A, et al. Involvement of chemokine receptors in breast cancer metastasis. Nature. 2001; 410(6824):50-56.

56. Wiley HE, Gonzalez EB, Maki W, Wu MT, Hwang ST. Expression of CC chemokine receptor-7 and regional lymph node metastasis of B16 murine melanoma. J Natl Cancer Inst. 2001;93(21):1638-1643.

57. Kawada K, et al. Chemokine receptor CXCR3 promotes colon cancer metastasis to lymph nodes. Oncogene. 2007;26(32):4679-4688.

58. Das S, et al. Tumor cell entry into the lymph node is controlled by CCL1 chemokine expressed by lymph node lymphatic sinuses. J Exp Med. 2013; 210(8):1509-1528.

59. Meier F, et al. Metastatic pathways and time courses in the orderly progression of cutaneous melanoma. BrJ Dermatol. 2002;147(1):62-70.

60. Kim M, et al. CXCR4 signaling regulates metastasis of chemoresistant melanoma cells by a lymphatic metastatic niche. Cancer Res. 2010;70(24):10411-10451.

61. Shields JD, Kourtis IC, Tomei AA, Roberts JM, Swartz MA. Induction of lymphoidlike stroma and immune escape by tumors that express the chemokine CCL21. Science. 2010;328(5979):749-752.

62. Lund AW, et al. VEGF-C promotes immune tolerance in B16 melanomas and cross-presentation of tumor antigen by lymph node lymphatics. Cell Reports. 2012;1(3):191-199.

63. Cohen JN, et al. Lymph node-resident lymphatic endothelial cells mediate peripheral tolerance via Aire-independent direct antigen presentation. J Exp Med. 2010;207(4):681-688.

64. Tewalt EF, Cohen JN, Rouhani SJ, Engelhard VH. Lymphatic endothelial cells - key players in regulation of tolerance and immunity. Frontiers Immunol. 2012;3:305.

65. Tewalt EF, et al. Lymphatic endothelial cells induce tolerance via PD-L1 and lack of costimulation leading to high-level PD-1 expression on CD8 T cells. Blood. 2012;120(24):4772-4782.

66. Huggenberger R, et al. An important role of lymphatic vessel activation in limiting acute inflammation. Blood. 2011;117(17):4667-4678.

67. Huggenberger R, Ullmann S, Proulx ST, Pytowski B, Alitalo K, Detmar M. Stimulation of lymphangiogenesis via VEGFR-3 inhibits chronic skin inflam- mation. J Exp Med. 2010;207(10):2255-2269.

68. Kajiya K, Sawane M, Huggenberger R, Detmar M. Activation of the VEGFR-3 pathway by VEGF-C attenuates UVB-induced edema formation and skin inflammation by promoting lymphangiogenesis. J Invest Dermatol. 2009;129(5):1292-1298.

69. Zhou Q, et al. Vascular endothelial growth factor C attenuates joint damage in chronic inflammatory arthritis by accelerating local lymphatic drainage in mice. Arthritis Rheum. 2011;63(8):2318-2328.

70. Christiansen A, Detmar M. Lymphangiogenesis and cancer. Genes Cancer. 2011;2(12):1146-1158.

71. Mumprecht $\mathrm{V}$, et al. In vivo imaging of inflammation- and tumor-induced lymph node lymphangiogenesis by immuno-positron emission tomography. Cancer Res. 2010;70(21):8842-8851.

72. Mumprecht V, Detmar M. In vivo imaging of lymph node lymphangiogenesis by immuno-positron emission tomography. Methods Mol Biol. 2013;961:129-140.

73. Zgraggen S, Ochsenbein AM, Detmar M. An important role of blood and lymphatic vessels in inflammation and allergy. J Allergy. 2013;2013:672381.

74. Mumprecht V, Roudnicky F, Detmar M. Inflammation-induced lymph node lymphangiogenesis is reversible. Am J Pathol. 2012;180(3):874-879.

75. Mumprecht V, Detmar M. Lymphangiogenesis and cancer metastasis. J Cell Mol Med. 2009; 13(8A):1405-1416

76. Vidal-Sicart S, Valdes Olmos R. Sentinel node mapping for breast cancer: current situation. J Oncol. 2012;2012:361341.

77. Proulx ST, Luciani P, Dieterich LC, Karaman S, Leroux JC, Detmar M. Expansion of the lymphatic vasculature in cancer and inflammation: New opportunities for in vivo imaging and drug delivery. J Control Release. 2013;172(2):550-557.

78. Marshall MV, et al. Near-infrared fluorescence imaging in humans with indocyanine green: a review and update. Open Surg Oncol J. 2010;2(2):12-25.

79. Proulx ST, et al. Non-invasive dynamic near-infrared imaging and quantification of vascular leakage in vivo. Angiogenesis. 2013;16(3):525-540

80. Proulx ST, et al. Use of a PEG-conjugated bright nearinfrared dye for functional imaging of rerouting of tumor lymphatic drainage after sentinel lymph node metastasis. Biomaterials. 2013;34(21):5128-5137.

81. Proulx ST, Detmar M. Molecular mechanisms and imaging of lymphatic metastasis. Exp Cell Res. 2013; 319(11):1611-1617

82. Leijte JA, van der Ploeg IM, Valdes Olmos RA, Nieweg OE, Horenblas S. Visualization of tumor blockage and rerouting of lymphatic drainage in penile cancer patients by use of SPECT/CT. J Nucl Med. 2009;50(3):364-367.

83. Nathanson SD, Mahan M. Sentinel lymph node pressure in breast cancer. Ann Surg Oncol. 2011; 18(13):3791-3796.

84. Blum KS, Proulx ST, Luciani P, Leroux JC, Detmar
M. Dynamics of lymphatic regeneration and flow patterns after lymph node dissection. Breast Cancer Res Treat. 2013;139(1):81-86

85. Planas-Paz L, Lammert E. Mechanical forces in lymphatic vascular development and disease. Cell Mol Life Sci. 2013;70(22):4341-4354.

86. Planas-Paz L, Strilic B, Goedecke A, Breier G, Fassler R, Lammert E. Mechanoinduction of lymph vessel expansion. EMBO J. 2012;31(4):788-804.

87. Van der Auwera I, et al. First international consensus on the methodology of lymphangiogenesis quantification in solid human tumours. Br J Cancer. 2006;95(12):1611-1625

88. Cueni LN, Detmar M. The lymphatic system in health and disease. Lymphat Res Biol. 2008;6(3-4):109-122.

89. Johnson LA, Prevo R, Clasper S, Jackson DG. Inflammation-induced uptake and degradation of the lymphatic endothelial hyaluronan receptor LYVE-1. J Biol Chem. 2007;282(46):33671-33680.

90. Mouta Carreira C, et al. LYVE-1 is not restricted to the lymph vessels: expression in normal liver blood sinusoids and down-regulation in human liver cancer and cirrhosis. Cancer Res. 2001;61(22):8079-8084.

91. Doeden K, Ma Z, Narasimhan B, Swetter SM, Detmar M, Dadras SS. Lymphatic invasion in cutaneous melanoma is associated with sentinel lymph node metastasis. J Cutan Pathol. 2009;36(7):772-780.

92. Tammela T, et al. Photodynamic ablation of lymphatic vessels and intralymphatic cancer cells prevents metastasis. Sci Transl Med. 2011;3(69):69ra11.

93. Alitalo AK, et al. VEGF-C and VEGF-D blockade inhibits inflammatory skin carcinogenesis. Cancer Res. 2013;73(14):4212-4221.

94. Tammela T, et al. Blocking VEGFR-3 suppresses angiogenic sprouting and vascular network formation. Nature. 2008;454(7204):656-660.

95. Kalin RE, Banziger-Tobler NE, Detmar M, Brandli AW. An in vivo chemical library screen in Xenopus tadpoles reveals novel pathways involved in angiogenesis and lymphangiogenesis. Blood. 2009; 114(5):1110-1122.

96. Sleeman JP, Nazarenko I, Thiele W. Do all roads lead to Rome? Routes to metastasis development. Int J Cancer. 2011;128(11):2511-2526.

97. Clasper $\mathrm{S}$, et al. A novel gene expression profile in lymphatics associated with tumor growth and nodal metastasis. Cancer Res. 2008;68(18):7293-7303.

98. Roesli C, Mumprecht V, Neri D, Detmar M. Identification of the surface-accessible, lineage-specific vascular proteome by two-dimensional peptide mapping. FASEB J. 2008;22(6):1933-1944.

99. Royston D, Jackson DG. Mechanisms of lymphatic metastasis in human colorectal adenocarcinoma. J Pathol. 2009;217(5):608-619.

100. Olszewski WL, Jain P, Ambujam G, Zaleska M, Cakala M, Gradalski T. Tissue fluid pressure and flow during pneumatic compression in lymphedema of lower limbs. Lymphatic Res Biol. 2011;9(2):77-83. 